\title{
Somatization Symptoms in a Primary Care Clinic at a Tertiary Hospital in Southern Nigeria
}

\author{
Chidi J. Okafor ${ }^{1^{*}}$, Bassey Edet $^{2 *}$, Udeme Asibong ${ }^{3 *}$ \\ ${ }^{1}$ Department of Psychiatry, College of Medical Sciences, University of Calabar, Calabar, Nigeria \\ ${ }^{2}$ Clinical Services Department, Federal Psychiatric Hospital Calabar, Calabar, Nigeria \\ ${ }^{3}$ Department of Family Medicine, College of Medical Sciences, University of Calabar, Calabar, Nigeria \\ Email:"dr chidi@yahoo.com
}

Received 23 August 2015; accepted 11 October 2015; published 14 October 2015

Copyright (C) 2015 by authors and Scientific Research Publishing Inc.

This work is licensed under the Creative Commons Attribution International License (CC BY). http://creativecommons.org/licenses/by/4.0/

(c) $\underset{\mathrm{EY}}{\mathrm{i}}$ Open Access

\section{Abstract}

Background: Somatic symptoms that are not attributable to organic pathology are common in general practice settings however, data in most parts of Africa including southern Nigeria are still scarce. The aim of our study was to examine such somatic symptoms reported by patients attending a primary care facility at a tertiary hospital in southern Nigeria as well as to motivate future research in this area. Method: The study was conducted at the General Out Patient Clinic (GOPC) of the University of Calabar Teaching Hospital (UCTH). It was a cross sectional study in which data were obtained from the case notes of 115 patients that presented in the clinic with somatic complaints which could not be attributed to organic pathology by their physicians. Results: While 46 of the patients were males, 69 were females. Their mean age was 37.7 (SD $=11.9$ ). Internal heat, crawling sensation, body pains and palpitations were the most prevalent symptoms reported by the patients. When the symptoms were sorted into various groups, the "subjective abnormal bodily sensation" was the most prevalent and far outnumbered the "pseudo neurological symptoms". Conclusion: A number of patients attending the GOPC of the UCTH seek consultations for medically unexplained somatic symptom. The most prevalent of these symptoms are internal heat and crawling sensations both of which are not stated in the criteria recognized by the International Classification of Diseases-version 10 (ICD-10) for the diagnosis of psychiatric disorders.

\section{Keywords}

Somatization, Medically Unexplained Somatic Symptom, Abnormal Bodily Sensations, Southern Nigeria

\footnotetext{
${ }^{*}$ The authors contribute equally.

${ }^{\#}$ Corresponding author.
}

How to cite this paper: Okafor, C.J., Edet, B. and Asibong, U. (2015) Somatization Symptoms in a Primary Care Clinic at a Tertiary Hospital in Southern Nigeria. Journal of Biosciences and Medicines, 3, 67-73. 


\section{Introduction}

A significant number of patients in primary care seek help for somatic symptoms, some of which are not adequately explained by a medical condition. Such symptoms are often referred to as Medically Unexplained Somatic Symptoms (MUSS) and may include common symptoms like aches, pains and fatigue. As reported in some studies, about $25 \%$ - 50\% of the symptoms encountered in primary healthcare have no evidence of physical disease [1] [2]. In the general population, somatic symptoms are also highly prevalent as rates of 30\% - 38\% have been reported for symptoms like aches and (or) pains [3] [4]. It has been shown that women are more likely than men to experience medically unexplained symptoms [5] [6] and this is partly due to an increased rate of anxiety and depression in women [5]. Many physicians feel helpless at the face of medically unexplained somatic symptoms and could institute inappropriate physical interventions [7]. Some of these interventions are often extensive, expensive and time consuming as such constituting a burden on both the patient and the healthcare system. This is worrisome especially when considered in the light of the fact that patients with such symptoms seek more of emotional support than the physical interventions often offered by the physicians [8].

Available data suggest that multiple factors including stressful life events can contribute to the development of medically unexplained somatic symptoms [9] [10]. The north London somatization study also lends support to the role of stressful life events in the aetiology of medically unexplained somatic symptoms [11].

The process of experiencing medically unexplained somatic symptom is referred to as somatization [12], a term implying an expression of psychological distress in the physical language of bodily symptoms [13]. It is one of the most frustrating problems in clinical practice for both the physicians-who find the patients very difficult to treat [14] and the patients - who are often dissatisfied with the quality of care they get from their physician [15] [16]. The difficulty encountered in treating somatizing Patients might stem from our limited knowledge/understanding of the condition. To improve our understanding and knowledge of somatization, this study was undertaken with an aim to identify the types of somatization symptoms that are prevalent amongst primary care clinic attendees in Calabar, a city in southern Nigeria.

\section{Method}

The study was conducted at the General Out Patients Clinic (GOPC) of the University of Calabar Teaching Hospital. The clinic also serves as a primary healthcare centre, taking both walk-in and referred patients. Clinics are conducted every Monday to Friday and family physicians attend to patients during the clinic sessions. The clinic was visited Monday to Friday during the study period which lasted for five weeks. The consulting family physicians were approached and the aim of the study explained to them. Their cooperation and assistance were solicited and these were granted. Inclusion criterion was presentation in the clinic with a single or combination of somatic complaints in which medical/organic causes have been excluded by the consulting physicians. Ethical committee's approval was obtained prior to the commencement of this study. Every clinic day, the consulting physicians were persuaded to look out for patients that presented with somatic complaints and to avail the researchers the case notes of such patients if no significant evidence of organic pathologies were found to account for their complaints. The clinic nurses assisted in retrieving such case notes from the consulting physicians immediately the patients consultations with the physician were over. The case notes were checked for the presenting complaints (as documented by the consulting physicians). The symptoms in the complaints were noted and this procedure was continued until 115 consecutive medical case notes that met the inclusion criterion were studied.

Data obtained were analyzed using Statistical Package for Social Sciences (SPSS) version 11. Chi square was used to assess differences between categorical variable and a confidence interval of $95 \%$ at significant level less than or equals to 0.05 was applied.

\section{Results}

The description of the socio demographic characteristics of the one hundred and fifteen patients whose medical case notes were studied is given in Table 1. Their age range was from 19 to 73 years with mean age of 37.7 and a standard deviation 11.9

Twenty-one (18.2\%) of the patients were young adults (less than 30 years) while three (2.6\%) of the patients were in their old age (70 years and above). 
Table 1. Socio-demographic characteristics of the 115 patients.

\begin{tabular}{|c|c|c|}
\hline Variables & Frequency & Percent (\%) \\
\hline \multicolumn{3}{|l|}{ Gender } \\
\hline Male & 46 & $40 \%$ \\
\hline Female & 69 & $60 \%$ \\
\hline \multicolumn{3}{|l|}{ Age (years) } \\
\hline$<30$ & 21 & $18.2 \%$ \\
\hline $30-49$ & 57 & $49.6 \%$ \\
\hline $50-69$ & 34 & $29.6 \%$ \\
\hline 70 and above & 3 & $2.6 \%$ \\
\hline \multicolumn{3}{|l|}{ Marital Status } \\
\hline Single & 46 & $40 \%$ \\
\hline Married & 56 & $48.7 \%$ \\
\hline Separated & 5 & $4.3 \%$ \\
\hline Widowed & 8 & $7 \%$ \\
\hline \multicolumn{3}{|l|}{ Work Status } \\
\hline Student & 16 & $13.9 \%$ \\
\hline Unemployed & 21 & $18.3 \%$ \\
\hline Employed & 78 & $67.8 \%$ \\
\hline
\end{tabular}

Table 2 shows the frequency distribution of their somatic symptoms. The symptoms are arranged in descending order of frequency of complaints. That is, a symptom with the highest number of complaint is shown first while the one with the least number of complaints is shown last.

As shown in Table 3, the subjective abnormal bodily sensation [17] was the most prevalent group of symptoms complained of by the patients. Table 4 shows the relationship between gender and the number of somatic complaints.

\section{Discussion}

\subsection{Socio-Demography of the Somatizing Patients}

About eighteen percent of the patients were young adults while $2.6 \%$ were elderly (very old) patients. The male to female ratio was 2:3. About a third of the patients (32.2\%) were consisted of students and the unemployed.

\subsection{Frequency of Symptoms}

A total of 19 medically unexplained somatic symptoms were identified and of these, "Internal heat/body heat (not fever)" was the most common complaint. This is in keeping with an earlier work by Okhomina and Ebie who found heat as the commonest symptom occurring in three-fifth of their subjects [18] but contrasts the finding in Ayonrinde's work where pain was recorded as the commonest symptom [19]. It should be noted that comparing this study with the previous ones will not give us a true picture of the situation. This is because these previous studies though hospital based, were carried out in psychiatric clinics unlike the present study which was conducted at a primary care clinic where patients with somatization are more likely to seek help [20].

In terms of group of symptoms, subjective abnormal bodily sensation was the most prevalent. The symptoms in this group far outnumbered the pseudo neurological symptoms (PNS). This finding might appear surprising because the PNS are the predominant symptom observed in hysteria/conversion disorders which were the forerunners of somatization disorder (a specific diagnostic entity recognized in the tenth revision of the International 
Table 2. Frequency distribution of the somatic complaints.

\begin{tabular}{cc}
\hline Symptoms & Frequency of Complaints \\
\hline Internal heat/Body heat & 23 \\
Sensation of insects/worm crawling on the body & 19 \\
Body pains & 18 \\
Heart beating fast (palpitation) & 15 \\
Frequent headache & 13 \\
Waist pain/Backache & 12 \\
Peppery feeling on the body & 8 \\
Lack of balance while walking & 6 \\
Numbness in parts of the body & 5 \\
Feeling like vomiting (nausea) & 5 \\
Difficulty in breathing & 3 \\
Stomach turns and makes noise & 2 \\
Body itching & 2 \\
Biting sensation on the body & 2 \\
Something blocking the throat & 2 \\
Feeling the heart cuts & 1 \\
Can't see properly & 1 \\
Heaviness in the head & 1 \\
Weak erection/sexual arousal & 1 \\
\hline
\end{tabular}

Table 3. Groups of symptoms in the somatic complaints.

\begin{tabular}{|c|c|c|}
\hline Group of Symptoms & Frequency of Complaints & Percent \\
\hline $\begin{array}{l}\text { Gastro Intestinal Tract (GIT) } \\
\text { Feel like vomiting (Nausea) } \\
\text { Stomach turns and makes noise }\end{array}$ & $\frac{\underline{5}}{2}$ & $\underline{4.9 \%}$ \\
\hline $\begin{array}{c}\text { Sexual/Genitourinary } \\
\text { Weak erection/Sexual arousal }\end{array}$ & $\frac{1}{1}$ & $\underline{0.7 \%}$ \\
\hline $\begin{array}{c}\text { Pains/Aches } \\
\text { Body pains } \\
\text { Frequent Headaches } \\
\text { Waist pains/Backaches }\end{array}$ & $\begin{array}{l}\frac{18}{13} \\
\underline{12} \\
\underline{43}\end{array}$ & $\underline{30.3 \%}$ \\
\hline $\begin{array}{c}\text { Subjective Abnormal Bodily Sensat } \\
\text { Body Heat/Internal Heat } \\
\text { Crawling Sensation on the body } \\
\text { Peppery Feeling } \\
\text { Body itching } \\
\text { Biting Sensation on the body } \\
\text { Heaviness in the Head }\end{array}$ & $\begin{array}{l}\frac{23}{19} \\
\frac{8}{2} \\
\frac{2}{2} \\
\underline{1} \\
\underline{58}\end{array}$ & $\underline{40.8 \%}$ \\
\hline $\begin{array}{l}\text { Cardio-Pulmonary } \\
\text { Heart beating fast (Palpitations) } \\
\text { Difficulty breathing } \\
\text { Feeling the heart cuts }\end{array}$ & $\begin{array}{c}15 \\
3 \\
1 \\
\underline{\mathbf{1 9}}\end{array}$ & $\underline{13.4 \%}$ \\
\hline $\begin{array}{l}\text { Pseudo-Neurological Symptoms } \\
\text { Lack of balance } \\
\text { Numbness } \\
\text { Something blocking the throat } \\
\text { Can't see properly }\end{array}$ & $\begin{array}{c}6 \\
5 \\
2 \\
1 \\
\mathbf{1 4}\end{array}$ & $\underline{9.9 \%}$ \\
\hline TOTAL & 142 & $100 \%$ \\
\hline
\end{tabular}


Table 4. Relationship between gender and number of somatic complaints.

\begin{tabular}{cccc}
\hline Number of Complaints & Male & Female & Total \\
\hline 1 & $21(65.6 \%)$ & $11(34.4 \%)$ & $32(100 \%)$ \\
2 & $14(36.8 \%)$ & $24(63.2 \%)$ & $38(100 \%)$ \\
3 and above & $11(24.4 \%)$ & $34(75.6 \%)$ & $45(100 \%)$ \\
TOTAL & $46(40 \%)$ & $69(60 \%)$ & $115(100 \%)$ \\
\hline
\end{tabular}

Chi Square $=13.5 ;$ Degree of freedom $=2 ; P=0.001$.

Classification of Diseases-ICD-10 [21]. The predominance of symptoms categorized as subjective abnormal bodily sensation in this study can be explained in line with observation of Prince who opined that people of mainly African cultures tend to employ physical complaints in order to be allowed to enter the sick role, since psychological complaints alone will not be considered sufficient grounds [22]. Prince went further to state that the most prevalent diseases in Africa present with temperature increase (for example, malaria) and crawling sensation (for example, Guinea-worm and Hook-worm). It will therefore not be surprising to see African patients formulate their psychological distress in terms of bodily complaints/symptoms of "heat" (as to mimic malaria) or "crawling sensations" (as to mimic worm infestations). This study found that the complaints of heat and crawling sensations were the most significant constituents of the category of the subjective abnormal bodily sensations accounting for over two-third of all the symptoms in that category. It is important to state that complaints involving this category of symptoms (subjective abnormal bodily sensations) are not included in the criteria for the diagnosis of somatization disorder-a diagnostic entity in the ICD-10.

\subsection{Gender and Somatization}

Another finding of this study was that women significantly reported more number of somatization symptoms than the males. This is in agreement with the studies conducted in the past by some researchers [23] [24]. However, it contrasts with a study by Ohaeri and Odejide who did not find gender difference in somatization amongst their subjects [25]. In Calabar (Efik) culture where the present study was conducted, males are regarded as stronger gender with higher threshold for discomfort and thus may not admit to discomfort until it becomes very severe. A typical calabar (Efik) man would not easily succumb to sickness and assume the sick role since nobody would take charge of his responsibilities. This tends to force him to cope with whatever sickness/discomfort he has until it becomes very severe or unbearable. For this reason, a calabar man is unlikely to complain of any symptom unless it is severe and unbearable. In addition to this, Hobbs et al had opined that when compared with males, there was a greater tendency for women to use somatic language to express psychological discomfort [26].

\section{Conclusion}

This study has shown that a number of patients attending the General Out Patient Clinic (GOPC) at the University of Calabar Teaching Hospital seek consultations for medically unexplained somatic symptoms and the most prevalent are "Heat", "Crawling sensation" and "Pain" symptoms. Since somatization implies an expression of psychological distress in bodily symptoms, it will be interesting to explore in future research whether or not somatizing patients in calabar have diagnosable psychological conditions that will require treatment by the psychiatrists. However, it is important to state that the somatization symptoms encountered in calabar (as revealed in this study) are mainly in the group of subjective abnormal bodily sensations and this group of symptoms are not explicitly stated in the symptom criteria recognised in the ICD-10 for the diagnosis of psychiatric disorders.

\section{Limitations}

The symptoms reviewed in this study were those presumed by the consulting family physicians to lack evidence of an organic pathology. The declaration of lack of evidence of organic pathology by the physicians may not mean that none exist since it is dependent on the skills and experience of the consulting physicians as well as availability of adequate facilities for investigations. Therefore, undiagnosed physical illness may have been responsible for some of the symptoms. 


\section{References}

[1] Peveler, R., Kilkenny, L. and Jubnibtg, A.L. (1997) Medically Unexplained Physical Symptoms in Primary Care: A Comparison of Self Report Screening Questionnaires and Clinical Opinion. Journal of Psychosomatic Research, 42, 245-252. http://dx.doi.org/10.1016/S0022-3999(96)00292-9

[2] Ring, A., Dowrick, C.F., Humphris, G.M., Davies, J. and Salmon, P. (2005) The Somatising Effect of Clinical Consultation: What Patients and Doctors Say and Do Not Say When Patients Present Medically Unexplained Physical Symptoms. Social Science \& Medicine, 61, 1505-1515. http://dx.doi.org/10.1016/j.socscimed.2005.03.014

[3] Dunnell, K. and Cartwright, A. (1972) Medicine Takers, Prescribers and Hoarders. Routledge, Kegan Paul, London.

[4] Hannay, D. (1978) Symptom Prevalence in the Community. Journal of the Royal College of General Practitioners, 28, 492-499.

[5] Kroenke, K. and Spitzer, R. (1998) Gender Differences in the Reporting of Physical and Somatoform Symptoms. Psychosomatic Medicine, 60, 150-155. http://dx.doi.org/10.1097/00006842-199803000-00006

[6] Kroenke, K. and Price, R. (1993) Symptoms in the Community: Prevalence, Classification and Psychiatric Comorbidity. Archives of Internal Medicine, 153, 2474-2480. http://dx.doi.org/10.1001/archinte.1993.00410210102011

[7] Page, L. and Wessely, S. (2003) Medically Unexplained Symptoms: Exacerbating Factors in the Doctor-Patient Encounter. Journal of the Royal Society of Medicine, 96, 223-227. http://dx.doi.org/10.1258/jrsm.96.5.223

[8] Ring, A., Dowrick, C., Humphris, G. and Salmon, P. (2004) Do Patients with Unexplained Physical Symptoms Pressurize General Practitioners for Somatic Treatment? A Qualitative Study. British Medical Journal, 328, 1057. http://dx.doi.org/10.1136/bmj.38057.622639.EE

[9] Craig, T., Boardman, A., Mills, K., Daly-Jones, O. and Drake, H. (1993) The South London Somatisation Study l: Longitudinal Course and the Influence of Early Life Experiences. British Journal of Psychiatry, 163, 579-588. http://dx.doi.org/10.1192/bjp.163.5.579

[10] Hotopf, M., Mayou, R., Wadsworth, M. and Wessely, S. (1999) Childhood Risk Factors for Adults with Medically Unexplained Symptoms: Results from a National Birth Cohort Study. American Journal of Psychiatry, 156, 17961800.

[11] Craig, T., Drake, H., Mills, K. and Boardman, A.P. (1994) The South London Somatisation Study II: Influence of Stressful Life Events, and Secondary Gain. British Journal of Psychiatry, 165, 248-258. http://dx.doi.org/10.1192/bjp.165.2.248

[12] Vikram, P. and Athula, S. (2006) Psychological Approaches to Somatization in Developing Countries. Advances in Psychiatric Treatments, 12, 56-62.

[13] Basky, A.J. and Kleiman, G.L. (1993) Overview: Hypochondriasis, Bodily Complaints and Somatic Styles. American Journal of Psychiatry, 140, 273-283.

[14] Ohaeri, J.U. and Olatawura, M.O. (1988) The Resilience of Somatisation Symptoms to Physical Methods of Treatment. Psychopathologie Africaine, XXII, 65-78.

[15] Deale, A. and Wessely, S. (2001) Patients Perception of Medical Care in Chronic Fatigue Syndrome. Social Science \& Medicine, 52, 1859-1864. http://dx.doi.org/10.1016/S0277-9536(00)00302-6

[16] Peters, S., Stanley, I., Rose, M. and Salmon, P. (1998) Patients with Medically Unexplained Symptoms: Sources of Patients Authority and Implications for Demands on Medical Care. Social Science \& Medicine, 46, 559-565. http://dx.doi.org/10.1016/S0277-9536(97)00200-1

[17] Morakinyo, O. (1983) A Phenomenological Study of Functional Abnormal Bodily Sensations in African Psychiatric patients. Psychopathologie Africaine, XIX, 343-355.

[18] Okhomina, F.O.A. and Ebie, J.C. (1973) Heat in the Head as a Psychiatric Symptom. In: Andonakoh, C.C., Ed., Procedings of the 4th Pan African Psychiatric Congress Workshop on Neurosis in Africa-Accra, New Times Corporation, Accra.

[19] Ayonrinde, A. (1977) Heat in the Head or Body: A Semantic Confusion. African Journal of Psychiatry, I, 59-63.

[20] Lipowski, Z.J. (1998) Somatization: The Concept and Its Clinical Application. American Journal of Psychiatry, 145, 1358-1368.

[21] World Health Organization (2006) The ICD-10 Classification of Mental \& Behavioural Disorders-Clinical Descriptions and Diagnostic Guidelines. WHO, Geneva.

[22] Prince, R. (1985) The Concept of Culture-Bound Syndrome: Anorexia Nervosa and Brain-Fag. Social Science and Medicine, 21, 197-203. http://dx.doi.org/10.1016/0277-9536(85)90089-9

[23] Andrian, F., Mark, O., Marc, G., Milton, F., Steven, S., Lantigua, R.A., et al. (2001) Medically Unexplained Symptoms in an Urban General Medicine Practice. Journal of Psychosomatics, 42, 261-268. 
http://dx.doi.org/10.1176/appi.psy.42.3.261

[24] Orenstein, H. (1989) Briquet Syndrome in Association with Depression and Panic: A Recoceptualization of Briquet Syndrome. American Journal of Psychiatry, 146, 334-338. http://dx.doi.org/10.1176/ajp.146.3.334

[25] Ohaeri, J.U. and Odejide, O.A. (1994) Somatization Symptoms among Patients Using Primary Healthcare Facilities in a Rural Community in Nigeria. American Journal of Psychiatry, 151, 728-731. http://dx.doi.org/10.1176/ajp.151.5.728

[26] Hobbs, P., Ballinger, C., Greenwood, C., Martins, B. and Mc Clure, A. (1984) Factor Analysis and Validation of the General Health Questionnaire in Men: A General Practice Survey. British Journal of Psychiatry, 144, 270-275.

http://dx.doi.org/10.1192/bjp.144.3.270 